\title{
Paeonol Suppresses Chondrosarcoma Metastasis through Up-Regulation of miR-141 by Modulating PKC $\delta$ and c-Src Signaling Pathway
}

Chi-Ting Horng ${ }^{1}$, Po-Chuen Shieh ${ }^{2}$, Tzu-Wei Tan ${ }^{3,4}$, Wei-Hung Yang ${ }^{5,6,7,8}$ and Chih-Hsin Tang ${ }^{3,4,9, *}$

1 Medical Education Center, Kaohsiung Armed Force General Hospital, Kaohsiung 802, Taiwan; E-Mail: mtc9042@yahoo.com.tw

2 Department of Pharmacy, Tajen University, Pingtung 907, Taiwan;

E-Mail: pochuen@mail.tajen.edu.tw

3 Graduate Institute of Basic Medical Science, China Medical University, Taichung 404, Taiwan; E-Mail: twtan@mail.cmu.edu.tw

4 Department of Pharmacology, School of Medicine, China Medical University, Taichung 404, Taiwan

5 Department of Orthopedic Surgery, Taichung Hospital, Ministry of Health and Welfare, Taichung 403, Taiwan; E-Mail: u766018@ms42.hinet.net

6 School of Chinese Medicine, China Medical University, Taichung 404, Taiwan

7 Department of Nursing, National Taichung University of Science and Technology, Taichung 403, Taiwan

8 Graduate Institute of Biotechnology, National Chung Hsing University, Taichung 402, Taiwan

9 Department of Biotechnology, College of Health Science, Asia University, Taichung 413, Taiwan

* Author to whom correspondence should be addressed; E-Mail: chtang@mail.cmu.edu.tw; Tel.: +886-4-2205-2121 (ext. 7726); Fax: +886-4-2233-3641.

Received: 16 April 2014; in revised form: 10 June 2014 / Accepted: 13 June 2014 / Published: 2 July 2014

\begin{abstract}
Chondrosarcoma, a primary malignant bone cancer, has potential for local invasion and distant metastasis, especially to the lungs. Patients diagnosed with it show poor prognosis. Paeonol (2'-hydroxy-4'-methoxyacetophenone), the main active compound of traditional Chinese remedy Paeonia lactiflora Pallas, exhibits anti-inflammatory and anti-tumor activity; whether paeonol regulates metastatic chondrosarcoma is largely unknown. Here, we find paeonol do not increase apoptosis. By contrast, at non-cytotoxic
\end{abstract}


concentrations, paeonol suppresses migration and invasion of chondrosarcoma cells. We also demonstrate paeonol enhancing miR-141 expression and miR-141 inhibitor reversing paeonol-inhibited cell motility; paeonol also reduces protein kinase $\mathrm{C}(\mathrm{PKC}) \delta$ and c-Src kinase activity. Since paeonol inhibits migration and invasion of human chondrosarcoma via up-regulation of miR-141 via PKC $\delta$ and c-Src pathways, it thus might be a novel anti-metastasis agent for treatment of metastatic chondrosarcoma.

Keywords: paeonol; chondrosarcoma; miR-141; metastasis

\section{Introduction}

Chondrosarcoma is derived from abnormal proliferation cartilage and accounts for about $26 \%$ of bone cancer. It usually occurs in males aged 10-80 years, most tumors appearing on the scapula, sternum, ribs, or pelvis [1,2]. In clinical practice, surgical resection remains the primary mode of therapy. Chondrosarcoma is reported as easily metastasizing to other organs: e.g., lung, liver [3,4]. Distant metastasis means poor prognosis and high incidence of fatality associated with this mesenchymal malignancy due to lack of an effective adjuvant therapy, making it important to explore novel remedies $[5,6]$.

Tumor invasion and metastasis are prominent biological traits of cancer cells [7]. Mortality in such cases chiefly results from spread to distant organs. The microRNAs (miRNAs) are small (about 22-nucleotides long), non-coding RNAs that can modulate targeted gene expression through either translational repression or mRNA cleavage. One miRNA can regulate a set of functionally relevant genes simultaneously, which may reinforce phenotypic change [8,9], miRNAs control gene expression by binding to complementary 3'UTR sequences of target mRNAs [10,11]. Deregulated expression of miRNAs is reported in human cancer and may affect multiple steps during metastasis [12]. Recent studies report miRNAs involved in metastatic progression [13,14]. On the other hand, they are cited for mediating chondrosarcoma progression and metastasis $[15,16]$, making miRNA a novel target for chondrosarcoma therapy.

Paeonol (2'-hydroxy-4'-methoxyacetophenone) is a key active compound of Paeonia lactiflora. Pallas is a traditional Chinese herb used in Asia and Europe to improve blood flow while suppressing expression of cyclooxygenase-2, nitric oxide synthase, as well as cell surface adhesion molecules TNF- $\alpha$ and IL-1 $\beta[17,18]$. This compound has anti-oxidant and anti-inflammatory activity and demonstrably suppresses tumor formation $[17,19,20]$; it is also reported as reducing metastasis of human cancer [21,22], yet its effects on metastasis by human chondrosarcoma cells are largely unknown. Here, we report paeonol inhibiting migration and invasion of human chondrosarcoma cells. In addition, up-regulation of the miR-141 through protein kinase $\mathrm{C}(\mathrm{PKC}) \delta$ and c-Src pathways are involved in paeonol-reduced cell motility. Data point to paeonol as an anti-metastatic agent for treatment of metastasic chondrosarcoma. 


\section{Results}

\subsection{Paeonol Does not Induce Cell Death in Human Chondrosarcoma}

Paeonol has been reported to promote cancer apoptosis [17,19,20]; we investigated whether paeonol induced cell death in human chondrosarcoma. Cytotoxic effect was examined by 3-(4,5-dimethylthiazol2-yl)-2,5-diphenyltetrazolium bromide (MTT) assay. Incubation of chondrosarcoma cells (JJ012 and SW1353) for $48 \mathrm{~h}$ did not affect viability (Figure 1A,B). We examined whether paeonol induced apoptosis in human chondrosarcoma cells by triphosphate nick-end labeling (TUNEL) staining and caspase 3 activity assay. Incubation of these cells with paeonol did not enhance TUNEL expression or caspase 3 activity (Figure 1C-F), indicating paeonol does not induce cell death in human chondrosarcoma. All subsequent experiments used this concentration range.

Figure 1. Paeonol does not induce apoptosis in human chondrosarcoma. (A,B) Cells were incubated with various concentrations of paeonol for $48 \mathrm{~h}$, viability examined by 3-(4,5-dimethylthiazol-2-yl)-2,5-diphenyltetrazolium bromide (MTT) assay $(n=5)$; (C,D) Cells were incubated with paeonol for $48 \mathrm{~h}$, triphosphate nick-end labeling (TUNEL)-positive ones were examined by flow cytometry $(n=6)$; and $(\mathbf{E}, \mathbf{F})$ Cells were incubated with paeonol for $24 \mathrm{~h}$, and caspase 3 activity was examined by caspase 3 ELISA kit $(n=5)$. Results are expressed as mean \pm S.E.M.

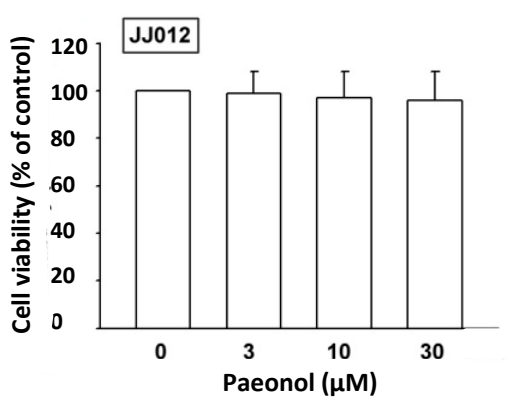

(A)

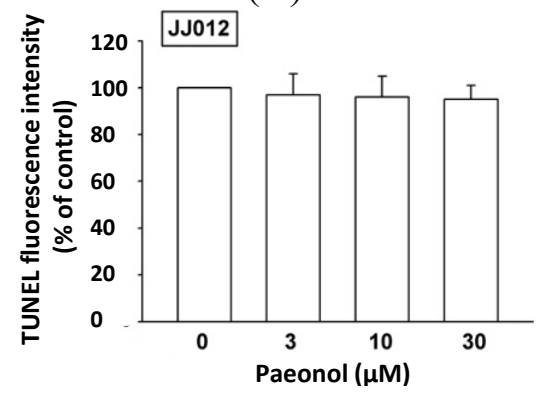

(C)

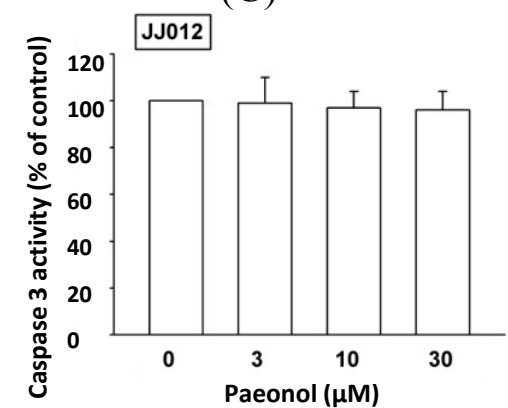

$(\mathbf{E})$

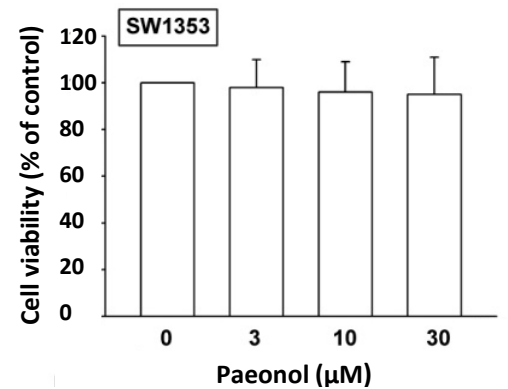

(B)

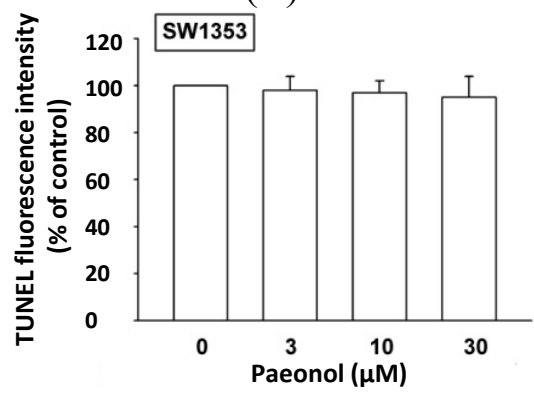

(D)

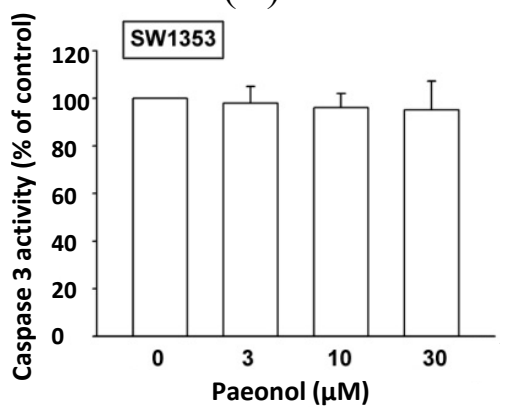

(F) 


\subsection{Paeonol Reduces Cell Migration, Wound-Healing Migration, and Cell Invasion}

Paeonol has been reported to reduce metastasis of human cancer cells [21,22]. We examined whether paeonol inhibits motility of chondrosarcoma cells. Incubation with paeonol $(3-30 \mu \mathrm{M})$ dramatically diminished migration in both chondrosarcoma cell lines (Figure 2A,B). In addition, wound-scratching assay confirmed that paeonol reduced wound healing activity in chondrosarcoma cells (Figure 2C,D). We also found paeonol reducing invasive ability via Matrigel basement membrane matrix (Figure 2E,F). Results indicated that paeonol reduces cell migration and invasion of human chondrosarcoma cells.

Figure 2. Paeonol inhibits migration and invasion of human chondrosarcoma. (A-F) Cells were incubated with various concentrations of paeonol for $24 \mathrm{~h}$; cell migration and invasion was examined through Transwell $(n=5)$, wound healing $(n=4)$, and invasion assays $(n=5)$. Results are expressed as the mean \pm S.E.M. *, $p<0.05$ compared with control.

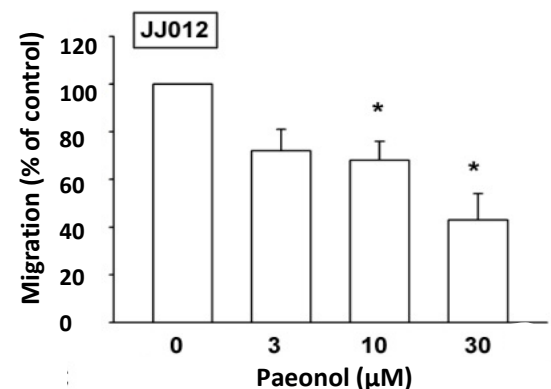

(A)

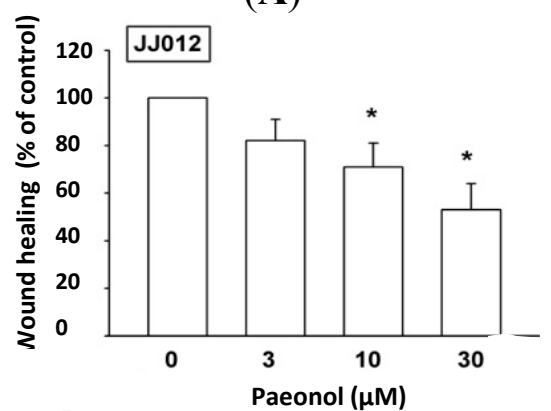

(C)

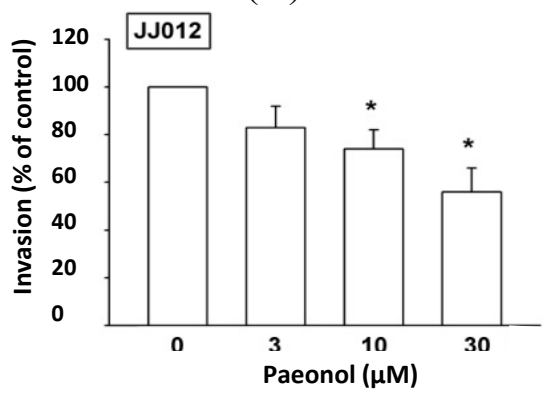

(E)

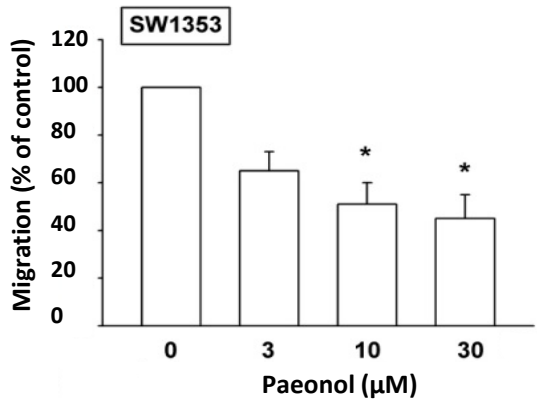

(B)

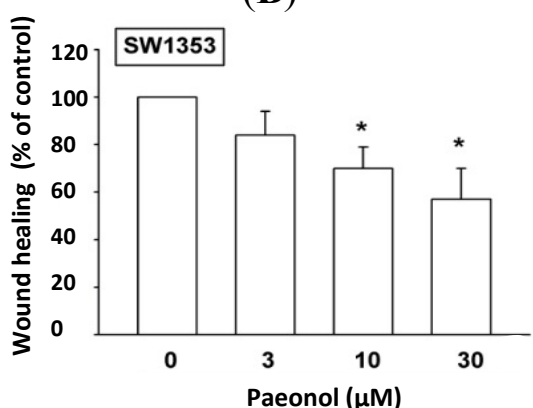

(D)

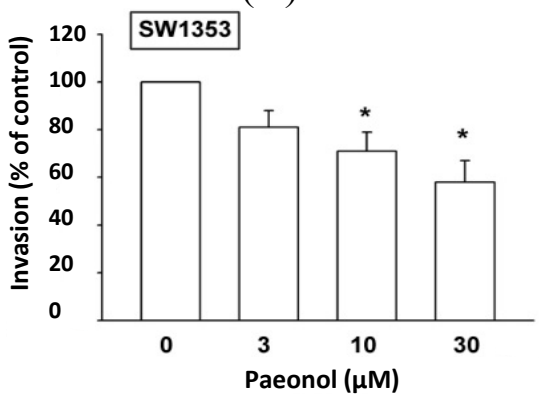

(F)

\subsection{Paeonol Reduces Motility in Chondrosarcoma Cells by Up-Regulation of miR-141 Expression}

The miRNAs have been reported as important regulators of cancer progression and metastasis [23], with miR-141 suggested to inhibit tumor migration and metastasis [24,25]. Up-regulation of miR-141 
is thus a novel strategy to suppress tumor motility. We tested paeonol's reduction of chondrosarcoma metastasis by modulating miR-141 expression to find incubation of chondrosarcoma cells with paeonol raising miR-141 expression in a concentration-dependent manner (Figure 3A,B). To affirm miR-141 involvement in paeonol-inhibited cell motility, miR-141 inhibitor transfection of cells reversed paeonol-suppressed cancer migration (Figure 3C,D). Data suggest paeonol reducing metastasis by up-regulating miR-141 expression.

Figure 3. Paeonol increases miR-141 expression in chondrosarcoma cells. (A,B) Cells were incubated with various concentrations of paeonol for $24 \mathrm{~h}$, miR-141 expression assessed by qPCR $(n=6)$; (C,D) Cells were transfected with miR-141 inhibitor for $24 \mathrm{~h}$, followed by stimulation with paeonol for $24 \mathrm{~h}$, cell migration examined by Transwell $(n=5)$. Results are expressed as mean \pm S.E.M. ${ }^{*}, p<0.05$ compared with control. \#, $p<0.05$ compared with paeonol-treated group.

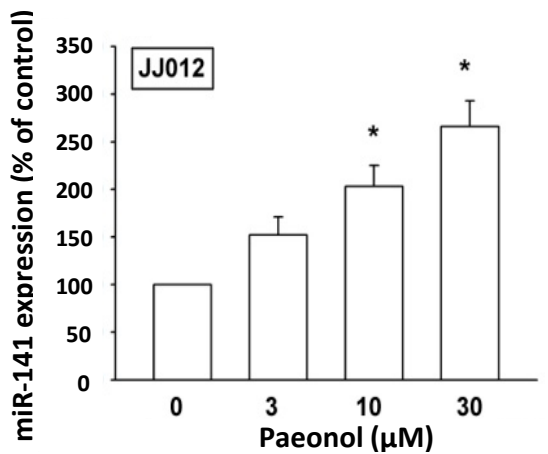

(A)

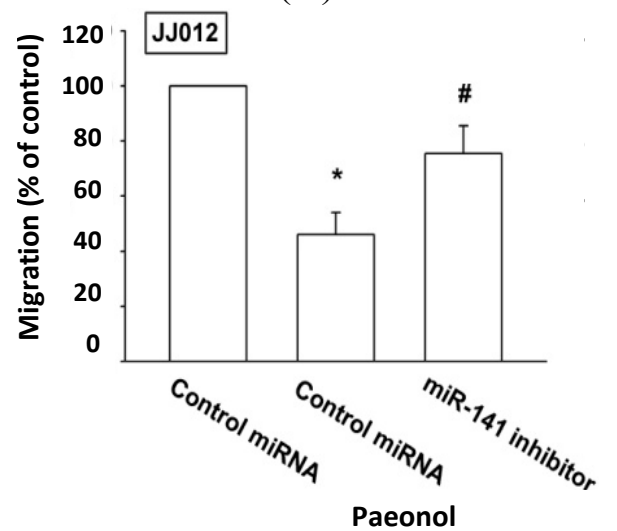

(C)

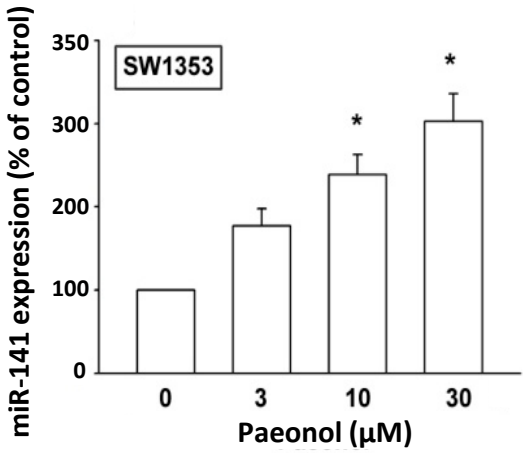

(B)

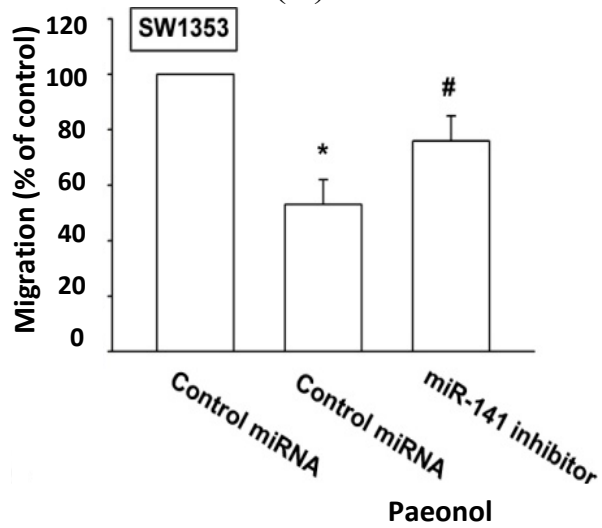

(D)

\subsection{Paeonol Reduces Activity of Protein Kinase C (PKC) $\delta$ and c-Src Signaling Pathways}

PKC $\delta$-dependent c-Src activation has been reported to mediate metastasis of human chondrosarcoma [26]. After the inhibitory effect of paeonol on cell migration was revealed, its effects on expression of the $\mathrm{PKC} \delta$ and c-Src pathways were investigated. Incubation of such cells with paeonol significantly decreased $\mathrm{PKC} \delta$ phosphorylation (Figure 4A,B), while paeonol suppressed $\mathrm{PKC} \delta$ kinase activity (Figure 4C,D). Likewise, c-Src phosphorylation and kinase activity were abolished by paeonol treatment in a concentration-dependent manner (Figure 5). Paeonol seems to act through a signaling pathway involving $\mathrm{PKC} \delta$ and c-Src to inhibit migration of chondrosarcoma cells. 
Figure 4. Protein kinase $\mathrm{C}(\mathrm{PKC}) \delta$ involved in paeonol response of human chondrosarcoma. Cells were incubated with various concentrations of paeonol for $24 \mathrm{~h}$; PKC $\delta$ phosphorylation and kinase activity was examined by western blot $(n=4)$ and PKC $\delta$ kinase activity kit $(n=6)$. Results are expressed as mean \pm S.E.M. *, $p<0.05$ compared with control.

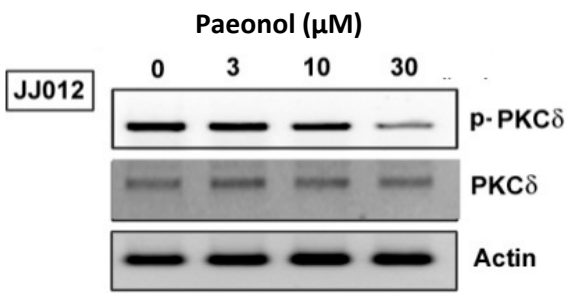

(A)

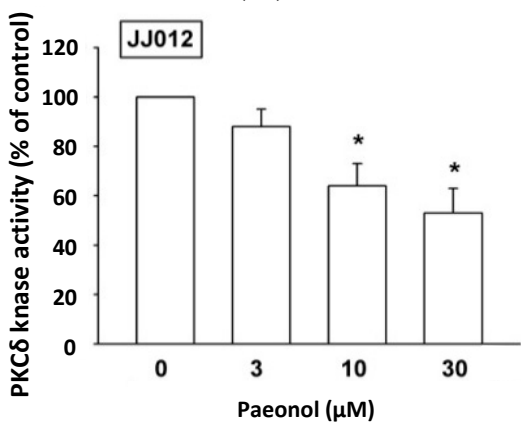

(C)

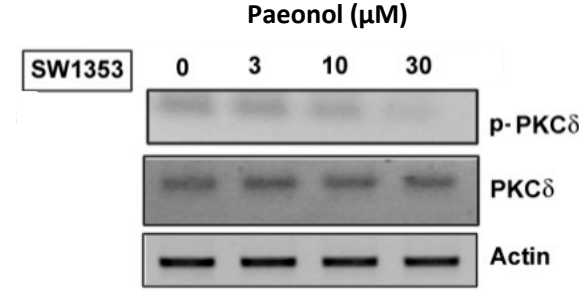

(B)

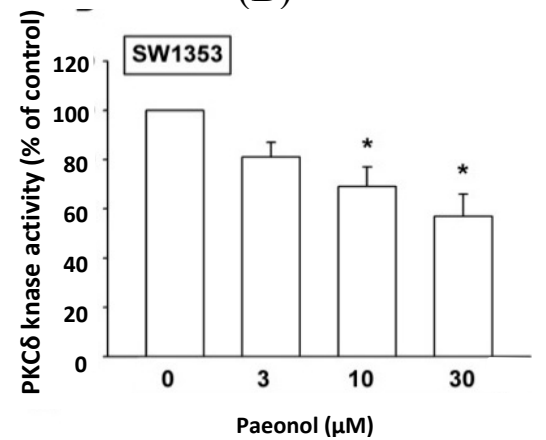

(D)

Figure 5. c-Src signaling pathway involved in paeonol response of chondrosarcoma. Cells were incubated with various paeonol concentrations for $24 \mathrm{~h}$, c-Src kinase activity and phosphorylation examined by western blot $(n=5)$ and c-Src kinase activity kit $(n=5)$. Results are expressed as mean \pm S.E.M. *,$p<0.05$ compared with control.

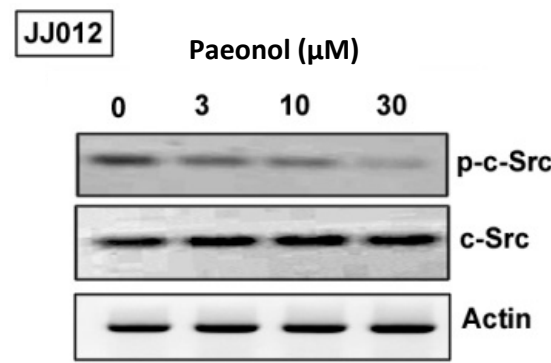

(A)

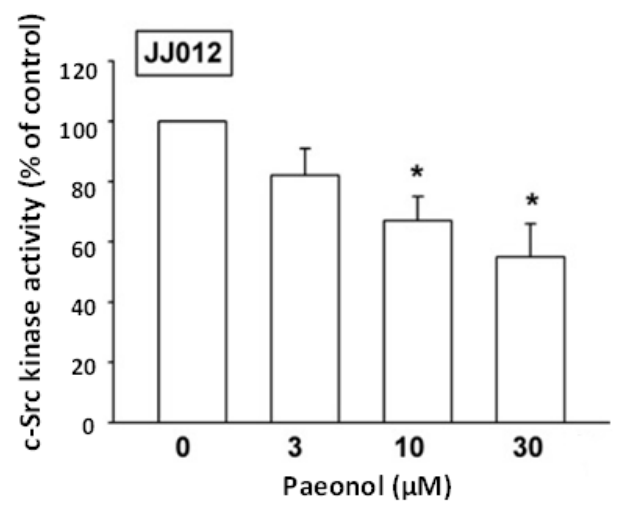

(C)

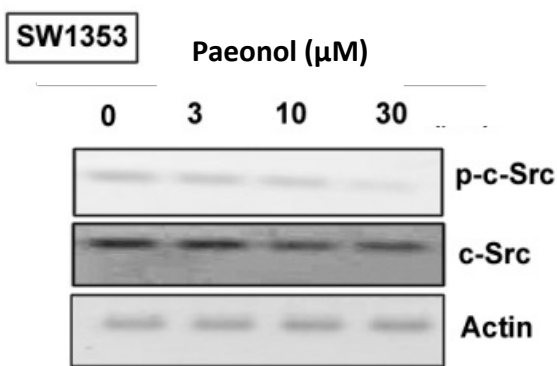

(B)

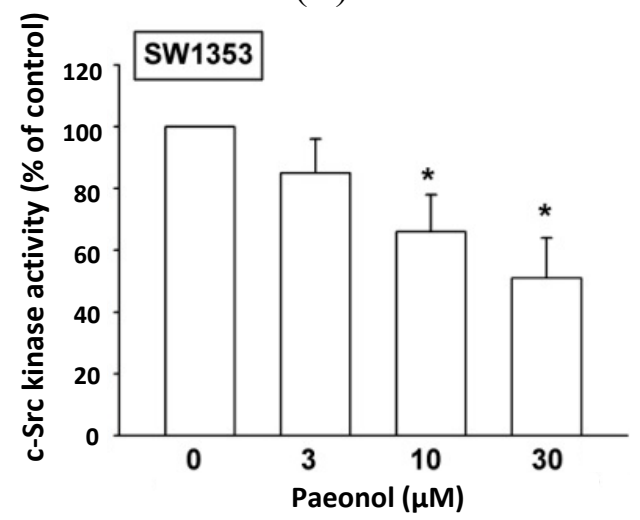

(D) 


\section{Discussion}

Chondrosarcoma, rare but deadly, accounts for nearly $26 \%$ of all bone cancers (the second most common type) [27]. Unlike other mesenchymal malignancies, such as osteosarcoma and Ewing's sarcoma, whose long-term survival dramatically increased with the advent of systemic chemotherapy, data indicate that chondrosarcoma shows a predilection for metastasis to lungs, meaning poor prognosis due to lack of effective adjuvant therapy [28,29]. It is thus crucial to develop effective adjuvant therapy to prevent chondrosarcoma metastasis. Paeonol has various biological activities, such as anti-aggregatory, antioxidant, anxiolytic-like and anti-inflammatory functions [17,30]; it reportedly inhibits tumor formation [20]. Increasing concentration of paeonol $(50 \mu \mathrm{M})$ slightly suppressed cell viability $(\sim 30 \%)$ in chondrosarcoma cells (data not shown), indicating that paeonol is not an antitumor agent for chondrosarcoma. Furthermore, paeonol does not have cytotoxic effects in normal chondrocytes and osteoblasts cells (3-30 $\mu \mathrm{M}$; data not shown). Paeonol has been described to inhibit metastatic potential of human cancer $[21,22]$. Yet anti-metastasic effects of paeonol on chondrosarcoma cells are mostly unknown. To simply look at the anti-metastasic effects of paeonol, we found that, at non-cytotoxic concentrations (3-30 $\mu \mathrm{M})$, paeonol reduced human chondrosarcoma cell motility. We found up-regulation of miR-141 through PKC $\delta$ and c-Src pathways involved in paeonol-reduced cancer migration. This study identified paeonol as a potential lead base, with good pharmacological properties, on anti-metastasic activity in human chondrosarcoma cells.

Newly identified, small noncoding miRNAs belong to a novel class of regulators that control gene expression by binding to complementary sequences in 3'UTRs of target mRNAs [31,32]. The miR-141 family is well known to inhibit migration and metastasis of human cancer [24,25,33], miR-141 as a negative regulator thereof. We hypothesized miR-141 mediating paeonol-inhibited cancer migration and found that paeonol increased miR-141 expression; transfection with miR-141 inhibitor rescued paeonol-reduced chondrosarcoma metastasis. Results indicate that paeonol reduces chondrosarcoma metastasis through up-regulation of miR-141 expression. miR-141 has been indicated to suppress the migration and invasion of hepatocellular carcinoma cells (HCC) by targeting Tiam1 [24]. Down-regulation of transmembrane-4-L-six-family-1 (TM4SF1) is involved in mR-141-inhibited pancreatic cancer cell invasion and migration [25]. In gastric cancer cells, miR-141 reduced cell motility by targeting hepatoma-derived growth factor (HDGF) [33]. Whether paeonol inhibited chondrosarcoma metastasis by up-regulating miR-141 through targeting these common molecules needs further examination.

$\mathrm{PKC} \delta$ is proven to mediate tumor migration and metastasis [34], while c-Src (PKC $\delta$-dependent) is involved in cancer migration and metastasis [31,35]. We found paeonol reducing $\mathrm{PKC} \delta$ phosphorylation and kinase activity in a dose-dependent manner, plus inhibiting c-Src phosphorylation and kinase activity in chondrosarcoma cells. Taken together, our results provide evidence that paeonol down-regulates cell motility in human chondrosarcoma cells via the $\mathrm{PKC} \delta / \mathrm{c}-\mathrm{Src}$ signaling pathway. In addition to cancer metastasis, a similar signal pathway has also been reported in the berberine reduced integrin expression and cell migration in chondrosarcoma cells, which inhibited the PKC $\delta$ and c-Src pathways [26], prostaglandin E2 regulated oral cancer migration, which involved PKC $\delta$-dependent c-Src pathway [36], and COX-2 promoted cancer metastasis, which mediated $\mathrm{PKC} \delta$ and $\mathrm{c}-\mathrm{Src}$ pathways [37]. Taken together, these results show that $\mathrm{PKC} \delta$-dependent $\mathrm{c}-\mathrm{Src}$ activation may have a role in treatment of cancer metastasis. 


\section{Experimental Section}

\subsection{Materials}

Paeonol was purchased from Wako Chemicals (Osaka, Japan); rabbit polyclonal antibodies specific for p-PKC $\delta, \operatorname{PKC} \delta$, p-c-Src, and c-Src from Biotechnology (Santa Cruz, CA, USA); miR-141 inhibitor and Lipofectamine 2000 from Life Technologies (Carlsbad, CA, USA); all other chemicals from Sigma-Aldrich (St. Louis, MO, USA).

\subsection{Cell Culture}

Human chondrosarcoma cell line JJ012 was kindly provided by the laboratory of Sean P. Scully (University of Miami School of Medicine, Miami, FL, USA) [14], cells cultured in Dulbecco's Modified Eagle's Medium (DMEM)/ $\alpha$-MEM supplemented with 10\% fetal bovine serum (FBS). Human chondrosarcoma cell line SW1353 was obtained from the American Type Culture Collection; these were cultured in DMEM supplemented with $10 \%$ FBS. All cells were maintained at $37{ }^{\circ} \mathrm{C}$ in humidified $5 \% \mathrm{CO}_{2}$ atmosphere.

\subsection{3-(4,5-Dimethylthiazol-2-yl)-2,5-diphenyltetrazolium Bromide (MTT) Assay}

Viability was rated with a MTT assay. Once treated with paeonol for $48 \mathrm{~h}$, cultures were washed with phosphate-buffered saline (PBS). Then, MTT $(0.5 \mathrm{mg} / \mathrm{mL})$ was added to each well, and the mixture was incubated at $37^{\circ} \mathrm{C}$ for $2 \mathrm{~h}$. To dissolve formazan crystals, culture medium was replaced with equal volume of DMSO. After shaking the mixture at room temperature for $10 \mathrm{~min}$, absorbance of each well was calculated at $550 \mathrm{~nm}$ by microplate reader (Bio-Tek, Winooski, VT, USA) [38].

\subsection{Triphosphate Nick-End Labeling (TUNEL) Assay}

Apoptosis was examined via terminal deoxynucleotidyl transferase-mediated deoxy-uridine TUNEL assay, using BD ApoAlert ${ }^{\mathrm{TM}}$ DNA Fragmentation Kit (BD Biosciences, Palo Alto, CA, USA). Cells incubated with paeonol for $48 \mathrm{~h}$ were trypsinized, fixed with $4 \%$ paraformaldehyde, and then permeabilized with $0.1 \%$ Triton-X-100 in $0.1 \%$ sodium citrate. After washing, cells were incubated with reaction mixture for $60 \mathrm{~min}$ at $37^{\circ} \mathrm{C}$, and stained cells were analyzed by flow cytometer.

\subsection{Caspase 3 Activity Assay}

This is based on the ability of active enzymes to cleave a chromophore from enzyme substrate Ac-DEVD-pNA. Lysates were prepared and incubated with anti-caspase 3. Immunocomplexes were incubated with peptide substrate in assay buffer (100 mM NaCl, $50 \mathrm{mM}$ 4-(2-hydroxyethyl)-1piperazine-ethanesulphonic acid (HEPES), $10 \mathrm{mM}$ dithiothreitol, $1 \mathrm{mM}$ EDTA, 10\% glycerol, 0.1\% 3-[(3-cholamidopropyl)-dimethylammonio]-1-propanesulfonate] (CHAPS), $\mathrm{pH} 7.4$ ) for $2 \mathrm{~h}$ at $37{ }^{\circ} \mathrm{C}$. Release of $p$-nitroaniline was monitored at $405 \mathrm{~nm}$; results signify percent change in activity compared to untreated control [39]. 


\subsection{Migration and Invasion Assay}

Migration assay used Transwell inserts (Costar, NY, USA; 8-mm pore size) in 24-well dishes. For invasion assay, filters were precoated with $30 \mu \mathrm{L}$ Matrigel basement membrane matrix (BD Biosciences, Bedford, MA, USA) for $30 \mathrm{~min}$. The following procedures were the same for both migration and invasion assays. After treatment with paeonol $(3,10$, or $30 \mu \mathrm{M})$ for $24 \mathrm{~h}$, cells were harvested and seeded to Transwell at $1 \times 10^{4}$ cells/well in serum-free medium, and then incubated for $24 \mathrm{~h}$ at $37{ }^{\circ} \mathrm{C}$ in $5 \% \mathrm{CO}_{2}$. Cells were fixed in $3.7 \%$ formaldehyde for $5 \mathrm{~min}$ and stained with $0.05 \%$ crystal violet in PBS for $15 \mathrm{~min}$. Cells on the upper side of filters were removed with cotton-tipped swabs, filters washed with PBS. Cells on the underside of filters were examined and counted under a microscope. Each experiment (performed in triplicate) was repeated at least three times.

\subsection{Wound-Healing Migration Assay}

For wound-healing migration assay, cells were seeded on 12 -well plates at a density of $1 \times 10^{5}$ cells/well in culture medium; $24 \mathrm{~h}$ after seeding, the confluent monolayer of culture was scratched with a fine pipette tip. Migration was visualized by microscope, the rate of wound closure observed at the time indicated.

\subsection{Quantitative Real-Time PCR (qRT-PCR) of $m R N A$ and $m i R N A$}

Total RNA was extracted from chondrosarcoma cells by a TRIzol kit (MDBio, Taipei, Taiwan). Reverse transcription proceeded with $1 \mu \mathrm{g}$ of total RNA and oligo (dT) primer [36,40]. Quantitative real-time PCR (qRT-PCR) analysis used Taqman ${ }^{\circledR}$ one-step PCR Master Mix (Applied Biosystems, Foster City, CA, USA); $100 \mathrm{ng}$ of total cDNA was added per $25 \mu \mathrm{L}$ reaction with sequence-specific primers and Taqman ${ }^{\circledR}$ probes. Sequences for target gene primers and probes were purchased commercially (GAPDH as internal control) (Applied Biosystems, Foster City, CA, USA), qPCR assays carried out in triplicate by StepOnePlus sequence detection system (Applied Biosystems, Foster City, CA, USA). Cycling conditions consisted of 10 -min polymerase activation at $95{ }^{\circ} \mathrm{C}$ followed by 40 cycles at $95{ }^{\circ} \mathrm{C}$ for $15 \mathrm{~s}$ and $60{ }^{\circ} \mathrm{C}$ for $60 \mathrm{~s}$. Threshold was set above non-template control background and within the linear phase of target gene amplification to calculate cycle number at which the transcript was detected (denoted $C_{\mathrm{t}}$ ) [12].

For miRNA assay, cDNA was synthesized from total RNA (100 ng) by TaqMan MicroRNA Reverse Transcription Kit (Applied Biosystems, Foster City, CA, USA); reactions were incubated first at $16{ }^{\circ} \mathrm{C}$ for $30 \mathrm{~min}$, then at $42{ }^{\circ} \mathrm{C}$ for $30 \mathrm{~min}$, followed by inactivation at $85{ }^{\circ} \mathrm{C}$ for $5 \mathrm{~min}$. Reactions were incubated in a 96 -well plate at $50{ }^{\circ} \mathrm{C}$ for $2 \mathrm{~min}, 95^{\circ} \mathrm{C}$ for $10 \mathrm{~min}$, followed by 30 cycles of $95{ }^{\circ} \mathrm{C}$ for $15 \mathrm{~s}$ and $60{ }^{\circ} \mathrm{C}$ for $1 \mathrm{~min}$ by StepOnePlus sequence detection system. Relative quantification of gene expression was performed with endogenous control gene (U6), threshold cycle $\left(C_{\mathrm{t}}\right)$ defined as fractional cycle number at which fluorescence passed the fixed threshold. Relative expression was calculated by comparative $C_{\mathrm{t}}$ method. 


\subsection{Western Blot Analysis}

Cellular lysates were prepared, proteins resolved by SDS-PAGE [16,40] and transferred to Immobilon polyvinylidene fluoride membranes. Blots were blocked with $4 \%$ bovine serum albumin for $1 \mathrm{~h}$ at room temperature, and then probed with rabbit anti-human antibodies against p-PKC $\delta$, PKC $\delta$, p-c-Src or c-Src (1:1000) for $1 \mathrm{~h}$ at room temperature (Santa Cruz Biotechnology; Santa Cruz, CA, USA). After three washes, blots were incubated with peroxidase-conjugated donkey anti-rabbit secondary antibody (1:1000) for $1 \mathrm{~h}$ at room temperature and visualized with enhanced chemiluminescence, using X-OMAT LS film (Eastman Kodak, Rochester, NY, USA).

\subsection{Kinase Activity Assay}

PKC $\delta$ and c-Src activity were gauged by PKC Kinase Activity Assay Kit (Assay Designs, Inc., Ann Arbor, MI, USA) and c-Src Kinase Activity Assay Kit (Abnova, Corp., Taipei, Taiwan). These kits are based on solid-phase ELISA using specific synthetic peptide as substrate for PKC $\delta$ or c-Src, and a polyclonal antibody that recognizes the phosphorylated form of substrate.

\subsection{Transfection with miRNA Inhibitor}

Chondrosarcoma cells were transfected with $100 \mathrm{nM}$ of miRNA inhibitor or control miRNA in serum- and antibiotic-free DMEM medium for $4 \mathrm{~h}$ using the Lipofectamine 2000 reagent $2 \mu \mathrm{L}$, then the transfected cells were allowed to rest for at least $24 \mathrm{~h}$. All stimulations were performed $24 \mathrm{~h}$ after transfection.

\subsection{Statistical Analysis}

Data are presented as mean \pm S.E.M. statistical analysis of both samples used a Student's $t$ test. Statistical comparisons of more than two groups were performed by one-way analysis of variance with Bonferroni's post-hoc test, and $p<0.05$ was considered significant.

\section{Conclusions}

It has been recommended that drugs made from natural products play a dominant role in pharmaceutical care. Natural products are vital sources of potential agents for chemotherapy and metastasis [41,42]. This study showed paeonol inhibiting migration and invasion by human chondrosarcoma cells, as well as up-regulation of miR-141 through PKC $\delta$ and c-Src pathways involved in paeonol-mediated effects. Evidence indicates paeonol as beneficial in reducing metastasis of human chondrosarcoma.

\section{Acknowledgments}

This study was supported by grant from the National Science Council of Taiwan (NSC 100-2320-B039-028-MY3; 102-2632-B-039-001-MY3; 102-2320-B-039-030-MY3), Taichung Hospital, Ministry of Health and Welfare (10321), Taiwan Ministry of Health and Welfare Clinical Trial and Research 
Center of Excellence (DOH102-TD-B-111-004), and CMU under the Aim for Top University Plan of the Ministry of Education, Taiwan.

\section{Author Contributions}

Conception and design of the work: C.-H.T. and C.-T.H.; Performed the experiments and analyzed the data: C.-T.H., P.-C.S., T.-W.T. and W.-H.Y.; Drafting the article: C.-T.H. and C.-H.T.

\section{Conflicts of Interest}

All authors have no financial or personal relationships with other people or organizations that could inappropriately influence our work.

\section{References}

1. Barnes, R.; Catto, M. Chondrosarcoma of bone. J. Bone Jt. Surg. Br. Vol. 1966, 48, 729-764.

2. Tang, C.H. Molecular mechanisms of chondrosarcoma metastasis. Biol. Med. 2012, 2, 92-98.

3. Yuan, J.; Dutton, C.M.; Scully, S.P. RNAi mediated MMP-1 silencing inhibits human chondrosarcoma invasion. J. Orthop. Res. 2005, 23, 1467-1474.

4. Chen, P.C.; Cheng, H.C.; Yang, S.F.; Lin, C.W.; Tang, C.H. The CCN family proteins: Modulators of bone development and novel targets in bone-associated tumors. Biomed. Res. Int. 2014, 2014, 437096.

5. Chen, J.C.; Fong, Y.C.; Tang, C.H. Novel strategies for the treatment of chondrosarcomas: Targeting integrins. Biomed. Res. Int. 2013, 2013, 396839.

6. Yin, M.C. Humans and cancer: An ongoing fight. Biol. Med. 2012, 2, 83.

7. Liu, S.S.; Chen, X.M.; Zheng, H.X.; Shi, S.L.; Li, Y. Knockdown of Rab5a expression decreases cancer cell motility and invasion through integrin-mediated signaling pathway. J. Biomed. Sci. 2011, $18,58$.

8. Lee, C.Y.; Huang, C.Y.; Chen, M.Y.; Lin, C.Y.; Hsu, H.C.; Tang, C.H. IL-8 increases integrin expression and cell motility in human chondrosarcoma cells. J. Cell. Biochem. 2011, 112, 2549-2557.

9. Tillhon, M.; Guaman Ortiz, L.M.; Lombardi, P.; Scovassi, A.I. Berberine: New perspectives for old remedies. Biochem. Pharmacol. 2012, 84, 1260-1267.

10. Diogo, C.V.; Machado, N.G.; Barbosa, I.A.; Serafim, T.L.; Burgeiro, A.; Oliveira, P.J. Berberine as a promising safe anti-cancer agent-Is there a role for mitochondria? Curr. Drug Targets 2011, 12, 850-859.

11. Tang, W.; Nakamura, Y.; Tsujimoto, M.; Sato, M.; Wang, X.; Kurozumi, K.; Nakahara, M.; Nakao, K.; Nakamura, M.; Mori, I. Heparanase: A key enzyme in invasion and metastasis of gastric carcinoma. Mod. Pathol. 2002, 15, 593-598.

12. Lerner, I.; Baraz, L.; Pikarsky, E.; Meirovitz, A.; Edovitsky, E.; Peretz, T.; Vlodavsky, I.; Elkin, M. Function of heparanase in prostate tumorigenesis: Potential for therapy. Clin. Cancer Res. 2008, 14, 668-676.

13. Maxhimer, J.B.; Pesce, C.E.; Stewart, R.A.; Gattuso, P.; Prinz, R.A.; Xu, X. Ductal carcinoma in situ of the breast and heparanase-1 expression: A molecular explanation for more aggressive subtypes. J. Am. Coll. Surg. 2005, 200, 328-335. 
14. Block, J.A.; Inerot, S.E.; Gitelis, S.; Kimura, J.H. Synthesis of chondrocytic keratan sulphate-containing proteoglycans by human chondrosarcoma cells in long-term cell culture. J. Bone Jt. Surg. Am. Vol. 1991, 73, 647-658.

15. Tong, K.M.; Chen, C.P.; Huang, K.C.; Shieh, D.C.; Cheng, H.C.; Tzeng, C.Y.; Chen, K.H.; Chiu, Y.C.; Tang, C.H. Adiponectin increases MMP-3 expression in human chondrocytes through AdipoR1 signaling pathway. J. Cell. Biochem. 2011, 112, 1431-1440.

16. Tang, C.H.; Hsu, C.J.; Fong, Y.C. The CCL5/CCR5 axis promotes interleukin-6 production in human synovial fibroblasts. Arthritis Rheumatol. 2010, 62, 3615-3624.

17. Chou, T.C. Anti-inflammatory and analgesic effects of paeonol in carrageenan-evoked thermal hyperalgesia. Br. J. Pharmacol. 2003, 139, 1146-1152.

18. Nizamutdinova, I.T.; Oh, H.M.; Min, Y.N.; Park, S.H.; Lee, M.J.; Kim, J.S.; Yean, M.H.; Kang, S.S.; Kim, Y.S.; Chang, K.C. Paeonol suppresses intercellular adhesion molecule-1 expression in tumor necrosis factor- $\alpha$-stimulated human umbilical vein endothelial cells by blocking p38, ERK and nuclear factor- $\kappa \mathrm{B}$ signaling pathways. Int. Immunopharmacol. 2007, 7, 343-350.

19. Seales, E.C.; Jurado, G.A.; Brunson, B.A.; Wakefield, J.K.; Frost, A.R.; Bellis, S.L. Hypersialylation of $\beta 1$ integrins, observed in colon adenocarcinoma, may contribute to cancer progression by up-regulating cell motility. Cancer Res. 2005, 65, 4645-4652.

20. Xu, S.P.; Sun, G.P.; Shen, Y.X.; Wei, W.; Peng, W.R.; Wang, H. Antiproliferation and apoptosis induction of paeonol in HepG2 cells. World J. Gastroenterol. 2007, 13, 250-256.

21. Lee, H.J.; Kim, S.A.; Jeong, S.J.; Han, I.; Jung, J.H.; Lee, E.O.; Zhu, S.; Chen, C.Y.; Kim, S.H. Paeonol oxime inhibits bFGF-induced angiogenesis and reduces VEGF levels in fibrosarcoma cells. PLoS One 2010, 5, e12358.

22. Kim, S.A.; Lee, H.J.; Ahn, K.S.; Lee, E.O.; Choi, S.H.; Jung, S.J.; Kim, J.Y.; Baek, N.; Kim, S.H. Paeonol exerts anti-angiogenic and anti-metastatic activities through downmodulation of Akt activation and inactivation of matrix metalloproteinases. Biol. Pharm. Bull. 2009, 32, 1142-1147.

23. Garzon, R.; Marcucci, G.; Croce, C.M. Targeting microRNAs in cancer: Rationale, strategies and challenges. Nat. Rev. Drug Discov. 2010, 9, 775-789.

24. Liu, Y.; Ding, Y.; Huang, J.; Wang, S.; Ni, W.; Guan, J.; Li, Q.; Zhang, Y.; Chen, B.; Chen, L. miR-141 suppresses the migration and invasion of HCC cells by targeting Tiam1. PLoS One 2014, 9, e88393.

25. Xu, L.; Li, Q.; Xu, D.; Wang, Q.; An, Y.; Du, Q.; Zhang, J.; Zhu, Y.; Miao, Y. hsa-miR-141 down-regulates TM4SF1 to inhibit pancreatic cancer cell invasion and migration. Int. J. Oncol. 2014, 44, 459-466.

26. Wu, C.M.; Li, T.M.; Tan, T.W.; Fong, Y.C.; Tang, C.H. Berberine reduces the metastasis of chondrosarcoma by modulating the $\alpha v \beta 3$ Integrin and the PKC $\delta$, c-Src, and AP-1 Signaling Pathways. Evid. Based Complement. Altern. Med. 2013, 2013, 423164.

27. Hsu, C.J.; Wu, M.H.; Chen, C.Y.; Tsai, C.H.; Hsu, H.C.; Tang, C.H. AMP-activated protein kinase activation mediates CCL3-induced cell migration and matrix metalloproteinase-2 expression in human chondrosarcoma. Cell Commun. Signal. 2013, 33, 68.

28. Wu, M.H.; Huang, C.Y.; Lin, J.A.; Wang, S.W.; Peng, C.Y.; Cheng, H.C.; Tang, C.H. Endothelin-1 promotes vascular endothelial growth factor-dependent angiogenesis in human chondrosarcoma cells. Oncogene 2014, 33, 1725-1735. 
29. Wu, M.H.; Chen, L.M.; Hsu, H.H.; Lin, J.A.; Lin, Y.M.; Tsai, F.J.; Tsai, C.H.; Huang, C.Y.; Tang, C.H. Endothelin-1 enhances cell migration through COX-2 up-regulation in human chondrosarcoma. Biochim. Biophys. Acta 2013, 1830, 3355-3364.

30. Mi, X.J.; Chen, S.W.; Wang, W.J.; Wang, R.; Zhang, Y.J.; Li, W.J.; Li, Y.L. Anxiolytic-like effect of paeonol in mice. Pharmacol. Biochem. Behav. 2005, 81, 683-687.

31. Yu, H.S.; Lin, T.H.; Tang, C.H. Bradykinin enhances cell migration in human prostate cancer cells through B2 receptor/PKCס/c-Src dependent signaling pathway. Prostate 2013, 73, 89-100.

32. Ozanne, B.W.; Spence, H.J.; McGarry, L.C.; Hennigan, R.F. Transcription factors control invasion: AP-1 the first among equals. Oncogene 2007, 26, 1-10.

33. Chen, B.; Huang, T.; Jiang, J.; Lv, L.; Li, H.; Xia, S. miR-141 suppresses proliferation and motility of gastric cancer cells by targeting HDGF. Mol. Cell. Biochem. 2014, 388, 211-218.

34. Lin, T.H.; Liu, H.H.; Tsai, T.H.; Chen, C.C.; Hsieh, T.F.; Lee, S.S.; Lee, Y.J.; Chen, W.C.; Tang, C.H. CCL2 increases $\alpha v \beta 1$ integrin expression and subsequently promotes prostate cancer migration. Biochim. Biophys. Acta 2013, 1830, 4917-4927.

35. Yang, S.F.; Chen, M.K.; Hsieh, Y.S.; Chung, T.T.; Hsieh, Y.H.; Lin, C.W.; Su, J.L.; Tsai, M.H.; Tang, C.H. Prostaglandin E2/EP1 signaling pathway enhances intercellular adhesion molecule 1 (ICAM-1) expression and cell motility in oral cancer cells. J. Biol. Chem. 2010, 285, 29808-29816.

36. Tzeng, H.E.; Tsai, C.H.; Chang, Z.L.; Su, C.M.; Wang, S.W.; Hwang, W.L.; Tang, C.H. Interleukin-6 induces vascular endothelial growth factor expression and promotes angiogenesis through apoptosis signal-regulating kinase 1 in human osteosarcoma. Biochem. Pharmacol. 2013, $85,531-540$.

37. Liu, J.F.; Fong, Y.C.; Chang, C.S.; Huang, C.Y.; Chen, H.T.; Yang, W.H.; Hsu, C.J.; Jeng, L.B.; Chen, C.Y.; Tang, C.H. Cyclooxygenase-2 enhances $\alpha 2 \beta 1$ integrin expression and cell migration via EP1 dependent signaling pathway in human chondrosarcoma cells. Mol. Cancer 2010, 9, 43.

38. Tsai, H.C.; Huang, C.Y.; Su, H.L.; Tang, C.H. CTGF increases drug resistance to paclitaxel by upregulating survivin expression in human osteosarcoma cells. Biochim. Biophys. Acta 2014, $1843,846-854$.

39. Tsai, H.C.; Huang, C.Y.; Su, H.L.; Tang, C.H. CCN2 enhances resistance to cisplatin-mediating cell apoptosis in human osteosarcoma. PLoS One 2014, 9, e90159.

40. Huang, C.Y.; Chen, S.Y.; Tsai, H.C.; Hsu, H.C.; Tang, C.H. Thrombin induces epidermal growth factor receptor transactivation and CCL2 expression in human osteoblasts. Arthritis Rheumatol. 2012, 64, 3344-3354.

41. Pezzuto, J.M. Plant-derived anticancer agents. Biochem. Pharmacol. 1997, 53, 121-133.

42. Hsu, S.C.; Chung, J.-G. Anticancer potential of emodin. BioMed 2012, 2, 108-116.

(C) 2014 by the authors; licensee MDPI, Basel, Switzerland. This article is an open access article distributed under the terms and conditions of the Creative Commons Attribution license (http://creativecommons.org/licenses/by/3.0/). 